\title{
Homogenization of resonant chiral metamaterials
}

\author{
Andrei Andryieuski, ${ }^{1, *}$ Christoph Menzel, ${ }^{2}$ Carsten Rockstuhl, ${ }^{2}$ \\ Radu Malureanu, ${ }^{1}$ Falk Lederer, ${ }^{2}$ and Andrei Lavrinenko ${ }^{1}$ \\ ${ }^{1}$ DTU Fotonik - Department of Photonics Engineering, \\ Technical University of Denmark, Ørsteds pl. 343, DK-2800 Kongens Lyngby, Denmark \\ ${ }^{2}$ Institute of Condensed Matter Theory and Solid State Optics, \\ Friedrich-Schiller-Universität Jena, Max-Wien-Platz 1, D-07743 Jena, Germany
}

(Dated: August 8, 2021)

\begin{abstract}
Homogenization of metamaterials is a crucial issue as it allows to describe their optical response in terms of effective wave parameters as e.g. propagation constants. In this paper we consider the possible homogenization of chiral metamaterials. We show that for meta-atoms of a certain size a critical density exists above which increasing coupling between neighboring meta-atoms prevails a reasonable homogenization. On the contrary, a dilution in excess will induce features reminiscent to photonic crystals likewise prevailing a homogenization. Based on Bloch mode dispersion we introduce an analytical criterion for performing the homogenization and a tool to predict the homogenization limit. We show that strong coupling between meta-atoms of chiral metamaterials may prevent their homogenization at all.

PACS numbers: 78.20.Ek, 81.05.Xj, 78.67.Pt, 42.25.Bs, 78.20.Bh
\end{abstract}

\section{INTRODUCTION}

At present chiral metamaterials (MMs) attract a great deal of interest of the scientific community since their potential to strongly affect the polarization state of light is promising for many applications. There are many manifestations of this potential of chiral MMs as giant optical activity $\underline{\underline{1}} \underline{\underline{3}}$, circular dichroism $\underline{\underline{4}} \underline{\underline{6}}$ and negative index of refraction ${ }^{7-12}$, which are either considerably enhanced in comparison with the naturally occurring phenomena or cannot be observed in nature at all.

By definition, the unit cell of a chiral material is not superimposable with its mirror image and any chiral material exhibits gyrotropy in general. However, a giant gyrotropy requires strong intrinsic resonances of the elementary constitutive components, i.e. the chiral "metaatoms". For non-resonant structures the optical rotary power per meta-atom layer is usually vanishingly small. Even for resonant structures the achievable polarization rotation is often below the requirements of applications. This limitation can be overcome by stacking of several functional layers. Conventionally, light propagation in multilayered MMs is described by assigning effective wave parameters (EPs) to this $\mathrm{MM}^{9,11,13}-17$, such as e.g. the propagation constant $k$ or the effective refractive index introduced as $n=k c / \omega$. Such EPs simplify the design and the description of functional MM devices. However, before assigning EPs it is of utmost importance to assure that the respective MM may be actually homogenized 18 .

When the mutual coupling between adjacent functional layers in a stack is strong, the convergence of e.g. the effective refractive index with the stack thickness towards its bulk value is poor $\underline{\underline{19}}$. Then, and this is a common situation, the EPs depend on the MM slab thickness. Such effective properties have very limited sense $\mathrm{e}^{20}$, since they cannot predict the response (for example, reflectance and transmittance) of a MM slab with an arbi- trary thickness. The EPs are very rarely identical for a single and an infinite number of monolayers, though designs exist exhibiting this property 21 . Near field coupling has been investigated for the constitutive elements of meta-atoms ${ }^{22-24}$, magnetic plasmon structures ${ }^{25,26}$ and chiral MM bi-layers27. The latter work [27] showed that the functional properties of the bi-layered MM with coupling between layers are different from the properties of a monolayer. However, up to our knowledge, the general analysis of the coupling effects on the EPs of chiral metamaterials has not been conducted yet.

The main purpose of this work is to provide a systematic and comprehensive analysis of the influence of the coupling effects on the EPs of chiral MMs and on the chiral MMs homogenization. We introduce an analytical criterion of homogeneity based on the dispersion relation of Bloch modes. We show that several chiral MMs can be homogenized and in such case there is an optimal MM period, or in other words, an optimal distance between MM layers. We also show that in other cases MMs cannot be homogenized in the spectral resonance domain despite of a weakening of the coupling among adjacent layers by increasing their separation.

We consider only effective wave parameters rather than any material $\mathrm{EPs}^{20,28}$. Hence, effects caused by the mesoscopic structure of MMs (nonlocality, spatial dispersion, see e.g. $[20,29]$ ) are not in the focus of this contribution. The aim is rather to show under which conditions a multilayered periodic chiral MM may be described as an effectively homogeneous medium, similar to the investigations performed for the fishnet $\mathrm{MM}^{30,31}$ and multilayered dielectric stack in ${ }^{32}$. To avoid any complications with the tensorial properties of homogenized materials, the direction of propagation is fixed along the axis pointing normal to the MM surface and the responses of the media are probed upon illumination with left- and right-circular polarized waves only. Because of normal incidence the 
eigenvalue problem reduces to a scalar problem.

After having introduced the methodology of the research in Sec. II, we analyze as an example the twistedcross $\mathrm{MM}^{1}$ with respect to its homogenization in Sec. III. Additionally we introduce an analytical homogenization criterion. As a counterexample for a structure that cannot be homogenized, the twisted split-ring resonator $\mathrm{MM}^{33}$ is considered in Sec. IV. The importance of coupling effects for chiral MMs is emphasized in the Sec. V. Mechanical analogy of the periodic metamaterial is also provided there. The Conclusions section sums up the work.

\section{METHODOLOGY}

For an adequate description of a MM as homogeneous several requirements have to be fulfilled.

i. The operating frequency must be below the first Bragg resonance $\Re(k a)<\pi$, where $a$ is the metaatom size in the direction of wave propagation. Otherwise, the MM constitutes a photonic crystal and its homogenization is meaningless ${ }^{18}$.

ii. The lateral MM periodic must be such that the propagation of nonzero diffraction orders is $\operatorname{suppressed}^{29}$, i.e., the transverse size of a unit cell is required to be smaller than half the wavelength.

iii. For each polarization state the light propagation must be governed by a single (fundamental) Bloch mode only ${ }^{29}$ having the smallest propagation losses.

iv. The incident light should predominantly couple to this Bloch mode 29,30 .

In general, situations may occur where light couples to a higher-order Bloch mode more efficiently than to the fundamental one. If this higher-order Bloch mode is more damped than the fundamental one, its impact becomes negligible above a certain thickness of the MM slab and EPs converge to those of the bulk. However, if the attenuation coefficients for all Bloch modes to which light can couple are comparable, then this leads to thicknessdependent EPs and results in the lack of homogeneity.

As the tool to analyze such questions we rely on the dispersion relation of Bloch modes. The respective dispersion diagrams provide comprehensive information about the eigenmodes that are sustained by the MM. The occurrence of a Bragg resonance can be extracted from the dispersion relation and occurs whenever $\Re(k a)=\pi$ holds. From the analysis of the dispersion of the imaginary part of the wavenumber, $\Im(k)$, we can evaluate the propagation losses. So it is easy to infer about requirements (iii) and (iv) - number of dominating modes and their losses by considering in detail the dispersion relation. Thus, the Bloch modes dispersion diagram is an efficient mean of
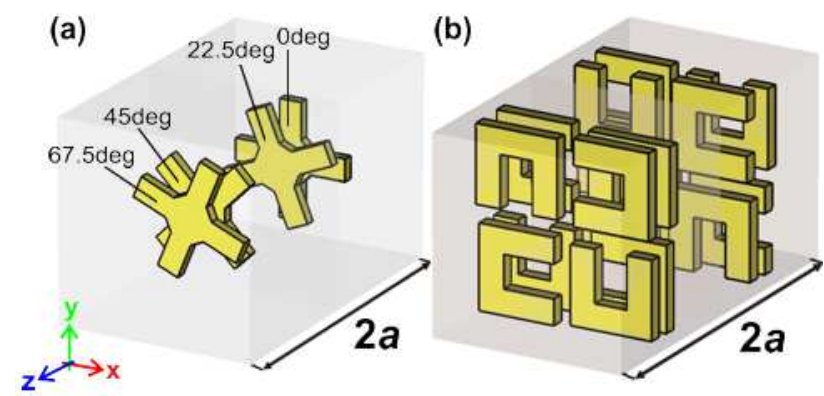

FIG. 1: (Color online). Metamaterial designs under consideration: TC (a) and TSRR (b). Wave propagation and metamaterial stacking direction is along $z$-axis.

MM homogeneity characterization. We would like to remark that in the case of chiral media with $C_{4}$-symmetry there are two fundamental modes: right and left circular polarized (RCP and LCP).

The chiral properties of a MM are determined through their effective refractive indices for the LCP and RCP waves the $n_{L}$ and $n_{R}$, respectively. The chirality parameter is given by $\kappa=\left(n_{R}-n_{L}\right) / 2$. Its real part $\Re(\kappa)$ describes the rotation of the polarization ellipse (or optical activity $)^{14}$ whereas the imaginary part $\Im(\kappa)$ governs the circular dichroism.

The first homogeneity requirement implies that a smaller lattice period $a$ in the propagation direction is beneficial. To check this statement we calculate the Bloch modes dispersion diagrams for both the twisted-cross metamaterials $\frac{1}{3}$ (Fig. 1 1 ) and twisted split-ring resonator metamaterials 33 (Fig. 10). The lateral sizes of their unit cells are such that the homogeneity requirement (ii) is satisfied in the whole frequency range of interest. These examples were only chosen for the sake of proving the applicability of our approach and constitute referential unit cells that are currently under investigation.

To simulate the optical response of MM slabs with various thicknesses we rely on the commercially available CST Microwave Studio software ${ }^{34}$ that implements the finite-integral method in the frequency domain. The thin slab EPs are retrieved from the complex reflection/transmission coefficients $\frac{15}{}$, which we refer to as the standard method. The thick slab EPs, which are close to bulk parameters, are restored from the spatial dependence of the wave field with the wave propagation retrieval method ${ }^{35,36}$. The Bloch dispersion diagram calculations are performed for infinite periodic media using a plane wave expansion method ${ }^{37}$ and taking into account the dispersion of the metal permittivity. Details of the calculation method can be found elsewhere $29,30,38$.

Comparing the spectral simulations of finite structures performed with the CST Microwave Studio with those done with the plane wave expansion method we observed a steady blue shift in spectra of about $20 \mathrm{THz}$ for the latter method. This shift that happens due to different numerical approaches is of minor importance for our conclusions. 


\section{TWISTED CROSS METAMATERIAL}

The twisted-cross (TC) $\mathrm{MM}^{1}$ (see Fig. 1a) is an example of a chiral MM that provides strong optical activity. Its meta-atoms consist of two golden crosses embedded into a dielectric. One cross is 22.5 degrees twisted with respect to the other. Lateral unit cell sizes are $a_{x}=a_{y}=500 \mathrm{~nm}$. The metallic crosses of thickness $25 \mathrm{~nm}$ are separated with a dielectric spacer of $37.5 \mathrm{~nm}$. The eigenfunctions of the TC are RCP and LCP because of the $C_{4}$-symmetry of the MM. For our investigation we use the parameters of Ref. [1]. However, in Ref. [1] only a monolayer is characterized. To access the bulk properties of such a MM we stack multiple monolayers as presented in Fig. 1a. The meta-atom size in the propagation direction $a$ is subject to variations. The crosses in each meta-atom are twisted by 45 degrees with respect to each other. Details can be seen in Fig. 1 1 where the angles of consecutive layers are indicated.

Note that, although the real unit cell size in propagation direction is $2 a$, the size $a$ is actually the length scale of importance because it denotes the periodic distance of consecutive functional layers. Since the optical response of the two parts of the unit cell are close to identical, we wish to regard this distance $a$ as the period. In fact, calculating the dispersion diagrams for the structure with the second double cross being identical to the first one, i.e. leading to a true period $a$, results qualitatively in identical characteristics. This holds except for the case of dense stacking, where the material becomes achiral. The Bragg resonances for the present structure are caused by the reflection of light at the consecutive layers of the unit cell rather than by the boundary between two unit cells. Bragg gaps due to the period $2 a$ are not observed. Therefore, we will call the parameter $a$ in the following the period of the structure.

To challenge the third requirement of the homogeneity we applied the standard retrieving (based on reflection/transmission) to thin MM slabs and the wave propagation method to thick MM slabs. The aim of such a retrieval with different methods was to check the convergence of the effective parameters. We studied MMs with two different center-to-center separations, the dense $(a=125 \mathrm{~nm})$ and the sparse stacking $(a=250 \mathrm{~nm})$. The thin-slab case consisted of 1-3 meta-atom layers for both MMs. For the thick slab EPs retrieval, 48 monolayers were stacked, thus mimicking the bulk material.

The structures exhibit completely different types of convergence with the slab thickness. For the dense stacking the convergence in the resonant region is very slow (Fig. 2a and b). So we suspect that the TC is not homogeneous there due to strong coupling between consecutive unit cells. The coupling can be reduced by separating the unit cells more. Indeed, for the sparse stacking, the effective refractive index of the thin slab quickly converges to the bulk values that are retrieved with the wave propagation retrieval method (Fig. 2r and d). This is a clear indication that such MMs might satisfy the homogeneity
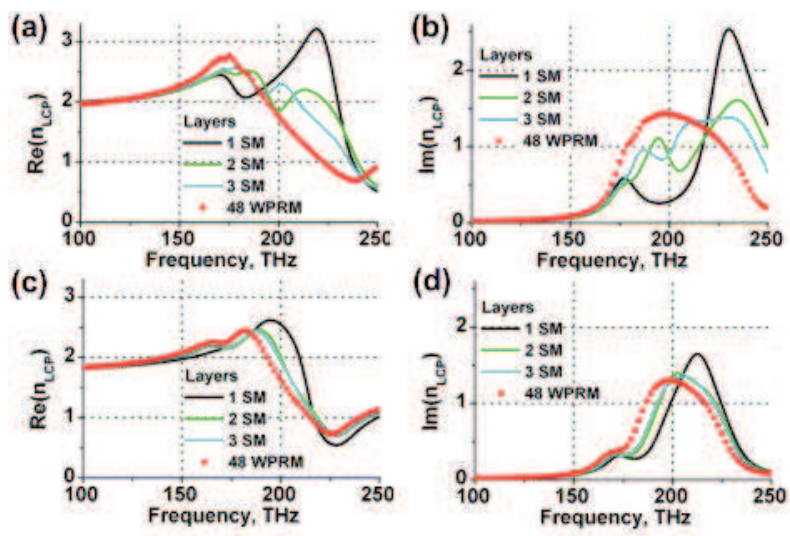

FIG. 2: (Color online). Effective refractive index of left circular polarized light of a twisted-cross metamaterial as a function of frequency and for different numbers of layers that form the MM. Dense stacking (period $2 a=250 \mathrm{~nm}) \Re\left(n_{L C P}\right)$ (a), $\Im\left(n_{L C P}\right)(\mathrm{b})$; sparse stacking (period $\left.2 a=500 \mathrm{~nm}\right) \Re\left(n_{L C P}\right)$ (c), $\Im\left(n_{L C P}\right)(\mathrm{d})$. Effective parameters are retrieved with the standard method $^{15}$ (SM) for one (black line), two (green/grey line) and three (cyan/light grey line) monolayers and with the wave propagation retrieval method ${ }^{36}$ (WPRM) for 48 layers (red/dark grey dots).

conditions.

To check the limits where TC homogenization fails we calculated the Bloch modes dispersion diagram for increasing separation, as shown in Fig. 3. It is clearly seen that for the dense stacking $(a=125 \mathrm{~nm})$ higher-order Bloch modes have a damping comparable with the fundamental modes in the resonant region (Fig. 33). Slow convergence of the EPs as seen in Fig. 2a, b, confirms that light couples to the higher-order Bloch modes, thus homogeneity requirement (iv) is violated.

For the sparse stacking case of $a=250 \mathrm{~nm}$ (Fig. 3 $\mathrm{b}$ ) the continuum of the higher-order Bloch modes is separated from the fundamental ones in terms of losses. The higher-order modes are much stronger damped than the fundamental ones. Thus one may expect that only the fundamental modes govern the light propagation. Moreover, the MM unit cells are still sub-wavelength (the resonant response occurs at frequencies far below the lowest order Bragg resonance), so all four requirements are satisfied, and the TC MM can be considered as a homogeneous.

Increasing the period to $a=400 \mathrm{~nm}$ we observe a new peak in $\Im(k)$ emerging at around $275 \mathrm{THz}$ and which is separated from the lowest resonance by a local minimum in the losses at $250 \mathrm{THz}$ (Fig. 3r.). The Bragg condition $\Re(k a)=\pi$ (the plot for $\Re(k a)$ not shown here) is satisfied between $250 \mathrm{THz}$ and $300 \mathrm{THz}$, therefore, this peak can be clearly identified as the first Bragg resonance. In the frequency region above the first Bragg resonance $(\Re(k a)>\pi)$ the properties of periodic arrangement of unit cells dominate and, according to requirement (i), homogenization fails. The frequency ranges where photonic crystal properties prevail are shadowed in Fig. 3 . 

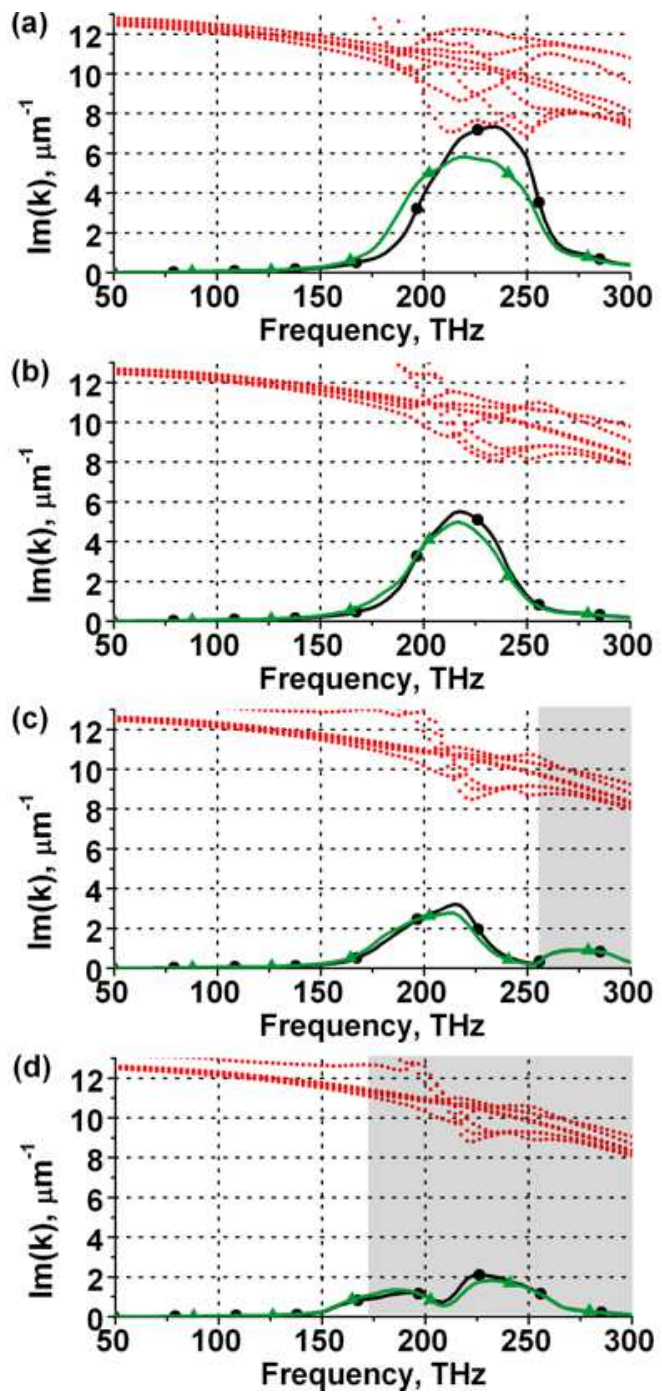

FIG. 3: (Color online). Bloch modes damping $\Im(k)$ dispersion spectra for twisted-cross MM for different separations: 125 nm (a), $250 \mathrm{~nm}$ (b), $400 \mathrm{~nm}$ (c), $500 \mathrm{~nm}$ (d). Fundamental modes are shown as black line with circles and green/grey line with triangles, all the higher-order Bloch modes are shown in red/dark grey dots. The frequency region above the first Bragg resonance is shadowed.

Increasing $a$ even further to $500 \mathrm{~nm}$ we face the situation where the first Bragg resonance overlaps with the intrinsic resonance of the meta-atoms at $220 \mathrm{THz}$ (Fig. $3 \mathrm{~d}$ ). Interestingly, the dip in the absorption spectrum at 210 $\mathrm{THz}$ appears instead of the peaks for all other separations (Fig. 3a $\mathrm{a}, \mathrm{b}$ and c). This resembles the phenomenon of electromagnetically induced transparency ${ }^{39.40}$, when two coupled oscillators with a small detuning show an absorption dip at the frequency, where the individual oscillators resonance is observed.

If we compare the magnitudes of mode losses extracted from Fig. 3, we conclude that losses of the higher-order Bloch modes remain almost constant while decreasing the unit cell size. On the contrary, the losses of the funda- mental modes increase considerably for smaller separation due to the increase of the metal filling fraction.

The brief summary of the presented results is that there exists a range of optimal longitudinal periods to achieve the best homogeneity for the twisted-cross MM, which is limited by the influence of the higher-order Bloch modes from one side and the Bragg resonance from the other side. To determine it we plot the difference $\Delta \Im(k a)=\min _{i \geq 3}\left(\Im\left(k_{i} a\right)\right)-\max \left(\Im\left(k_{1} a, k_{2} a\right)\right)$ between the attenuation constants of the least damped higher-order Bloch mode and the most damped fundamental mode (Fig. 4a). We suggest to neglect higherorder Bloch modes in the consideration if the difference in damping per unit cell is $\Delta \Im(k a) \geq 0.5$. In this case the intensity of the higher-order modes will be attenuated $e$ times more over the period $a$, compared to the fundamental modes. In the resonant region between 200 and $250 \mathrm{THz}$ the homogeneity condition $\Delta \Im(k a)>0.5$ is satisfied for $a \geq 200 \mathrm{~nm}$ (Fig. 田). Outside the resonant region this condition is much more moderate and the TC can be homogenized for smaller values of $a$.

The dependence of the minimum of the damping difference $\min \Delta \Im(k a)$ as a function of the period $a$ is shown in Fig. 4b. Surprisingly, this dependence is not only monotonously rising but also close to linear. This fact points out the predictive potential of the used methodology. Thus, it suffices to calculate the Bloch modes for only two periods in order to approximately predict the homogenization limit for the designed metamaterial.

We also checked the homogenization criterion $\Delta \Im(k a)>0.5$ for the split cube in cage negative index $\mathrm{MM}^{21}$. This MM shows very fast convergence of the EPs with the number of monolayers - the refractive index is the same from one monolayer to the infinite number of monolayers. In the whole frequency range of interest higher-order Bloch modes are well separated from the fundamental ones $(\Delta \Im(k a)>1.5)$ that explains why the EPs of the split-cube in cage do not depend on the slab thickness.

\section{TWISTED SPLIT-RING RESONATOR METAMATERIAL}

It is natural to make a hypothesis that for some metamaterial designs with strong coupling between consecutive layers, the MM cannot be homogenized in the resonance region at all. In other words, $\Delta \Im(k a)<0.5$ even for the large separation of meta-atoms where homogenization is prevented by the appearance of Bragg resonances. To check this hypothesis we investigated the twisted split-ring resonator (TSRR) $\mathrm{MM}^{33}$ (see Fig. 1). It consists of the golden U-shaped split-ring resonators embedded in a dielectric. The in-plane lattice constants are $a_{x}=a_{y}=885 \mathrm{~nm}$. The split rings of thickness 60 $\mathrm{nm}$ are separated by a dielectric spacer of $85 \mathrm{~nm}$. All the geometrical and material parameters are taken as in Ref.[33] except the period $a$. 

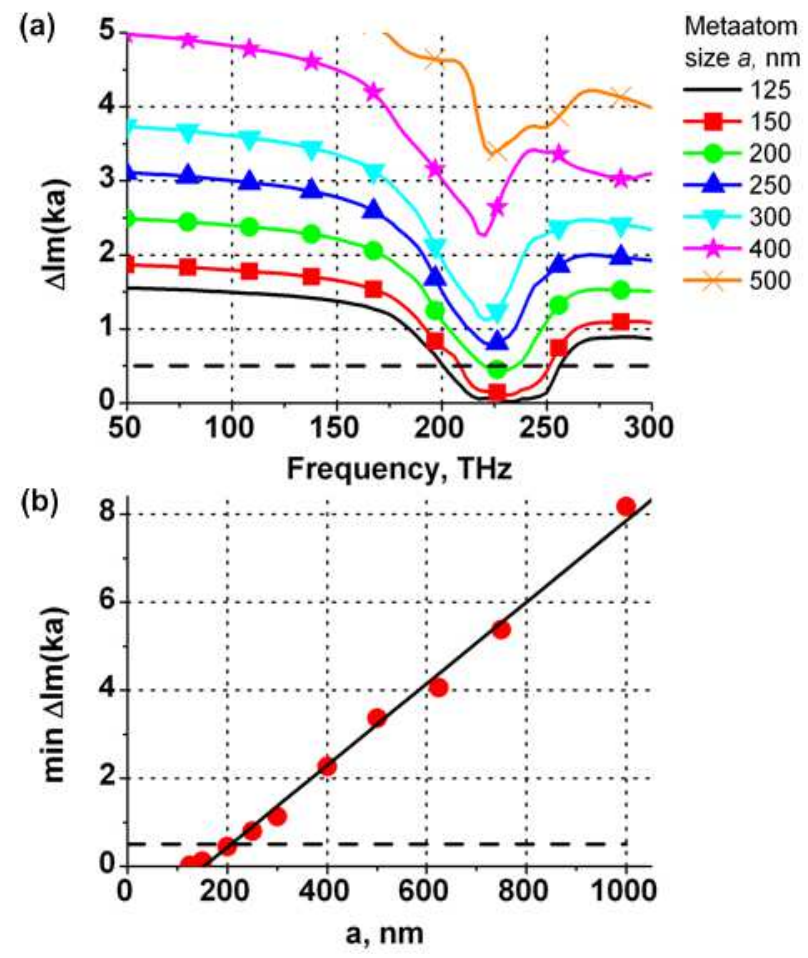

FIG. 4: (Color online). (a) The difference of the propagation constants $\Delta \Im(k a)=\min _{i \geq 3}\left[\Im\left(k_{i} a\right)\right]-\max \left[\Im\left(k_{1} a, k_{2} a\right)\right]$ of the least damped higher-order Bloch mode and the most damped fundamental mode for various unit cell sizes $a$ : $125 \mathrm{~nm}$ (black line), $150 \mathrm{~nm}$ (red line with squares), $200 \mathrm{~nm}$ (green line with circles), $250 \mathrm{~nm}$ (blue line with triangles up), $300 \mathrm{~nm}$ (orange line with triangles down), $400 \mathrm{~nm}$ (magenta line with stars), $500 \mathrm{~nm}$ (brown line with crosses). (b) The minimum of $\min \Delta \Im(k a)$ in (a) as a function of the period (red circles). The straight line represents a linear fit of min $\Delta \Im(k a)$ on $a$. The dashed line corresponds to the homogenization limit $\Delta \Im(k a)=0.5$.

We calculated the Bloch modes dispersion diagram for three center-to-center separations of the monolayers: $a=290 \mathrm{~nm}, a=450 \mathrm{~nm}$ and $a=600 \mathrm{~nm}$. One of the fundamental modes designated by the black solid line in Fig. [5 fails to satisfy the homogeneity requirements even for the sparsest stacking at $a=600 \mathrm{~nm}$. Its losses are higher than those for a few of the higher-order modes. The other fundamental mode (Fig. 5 green line) exhibits a comparable $\Im(k a)$ with one of the higher-order Bloch mode for $a=290 \mathrm{~nm}$ and $a=450 \mathrm{~nm}$. For the sparsest stacking $a=600 \mathrm{~nm}$, this fundamental mode experiences a slightly lower, but still unsufficient for homogenization, damping, since $\Delta(\Im(k a))=0.09<0.5$. A further increase of the lattice period to $a=600 \mathrm{~nm}$ shifts the real part of the wavevector into the first band gap $(\Re(k a)=\pi)$ for frequencies above $80 \mathrm{THz}$, so the requirement (i) is not met. We would hardly term a metamaterial homogeneous if only one eigenwave (for example, $\mathrm{RCP}$ ) fulfills the homogenization requirements. We may conclude that the TSRR does not satisfy requirement (iii)
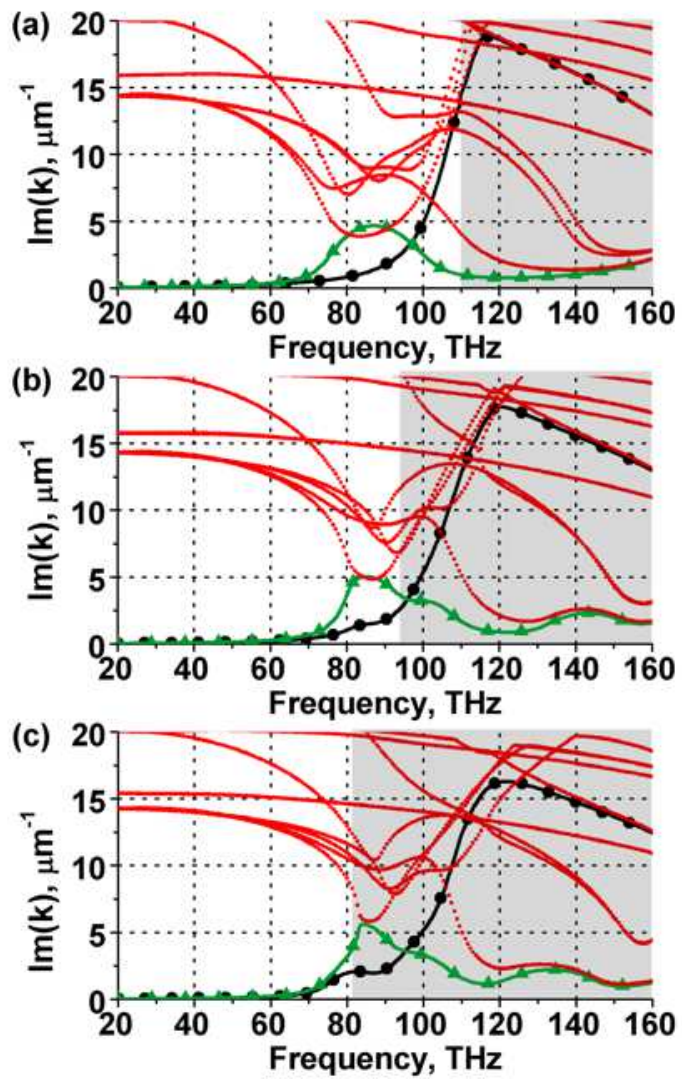

FIG. 5: (Color online). The loss spectrum for TSRR MM for different periods: $290 \mathrm{~nm}$ (a), $450 \mathrm{~nm}$ (b), $600 \mathrm{~nm}$ (c). Fundamental modes are shown as black lines with dots and green/grey lines with triangles, all higher-order Bloch modes are shown as red/dark grey dots. The frequency region above the first Bragg resonance is shadowed.

around the resonance frequency for any period. This is caused by the strong coupling between its meta-atoms. Thus, we prove our hypothesis that there exist MMs that cannot be considered homogeneous at the resonance frequency region.

It is important to mention that such MM keeps its optical functionality, i.e. affecting the state of polarization. However, one must not extrapolate the properties of a single or a few functional layers towards a stack of more functional layers.

\section{DISCUSSION}

The homogeneity criterion (i) requires a unit cell/wavelength ratio to be as small as possible. However, in this case, as shown above, in the resonance region requirements (iii) (single Bloch mode) and (iv) (efficient coupling of light to this specific mode) are frequently not met. When decreasing the unit cell size $a$, the fundamental modes' damping increases (see Fig. 3), so sooner or later it becomes comparable with the higher-order Bloch modes' damping. The higher-order Bloch modes then 
(a)
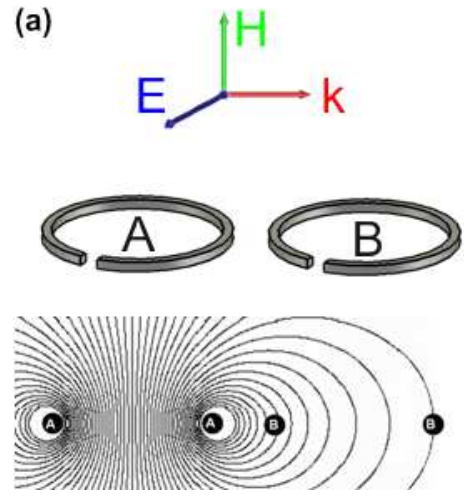

(b)
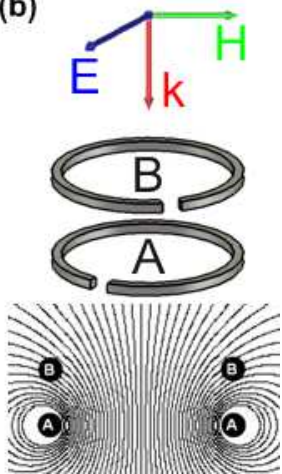

FIG. 6: The sketch of the split-ring resonators stacking in the negative-permeability configuration (a) and in the chiral configuration (b). Arrows represent the direction of the wave propagation, electric and magnetic field vectors. Black lines in the lower pictures represent the cross-section view of the lines of the magnetic field created by SR A and penetrating SR B.

have a comparable impact on the wave propagation in a MM slab as a direct consequence of the strong interaction of the meta-atom monolayers.

This conclusion, which is basically applicable to any MM, is extremely important for chiral MMs where the coupling of monolayers is usually strong. For example, for a negative-permeability configuration of the split-ring (SR) resonator metamaterial in the side-coupled arrangement, where the wavevector lies in the SR plane, SRs are weakly coupled due to the mutual inductance (magnetic field penetration) (see the sketch in Fig. 6a). The induced current in SR A creates a magnetic field that penetrates SR B. However, the number of the field lines penetrating SR B is not very large. On the contrary, for a chiral configuration, when the stacking direction is perpendicular to the SR plane (see Fig. 6b), the magnetic field of SR A strongly penetrates SR B.

Most chiral metamaterials consist of split-ring resonators or gammadions, where the meta-atoms are stacked similarly as in Fig. 6b. The other configuration, where the magnetic field of one meta-atom penetrates weakly into the other one, corresponds to the twistedcross MM. It is basically an extension of the cut-wire pairs towards $C_{4}$-symmetry. The currents excited in the double crosses create the magnetic field perpendicular to the stacking direction and are thus weakly penetrating into the adjacent monolayer (similar as in Fig. 6a).

Historically, e.g. by Newton, Bernoulli, Lagrange and others, wave phenomena were frequently illustrated by taking advantage of mechanical analogies. The historical overview of a periodic medium description with a springball system can be found in Brillouin's book ${ }^{41}$. We likewise employ a mechanical spring-ball system to illustrate the coupling effect in our MM system, see Fig. 7 .

The host dielectric medium is represented by chains of small balls and springs. Meta-atoms are represented in Fig. 7 as massive oscillators having their own reso-
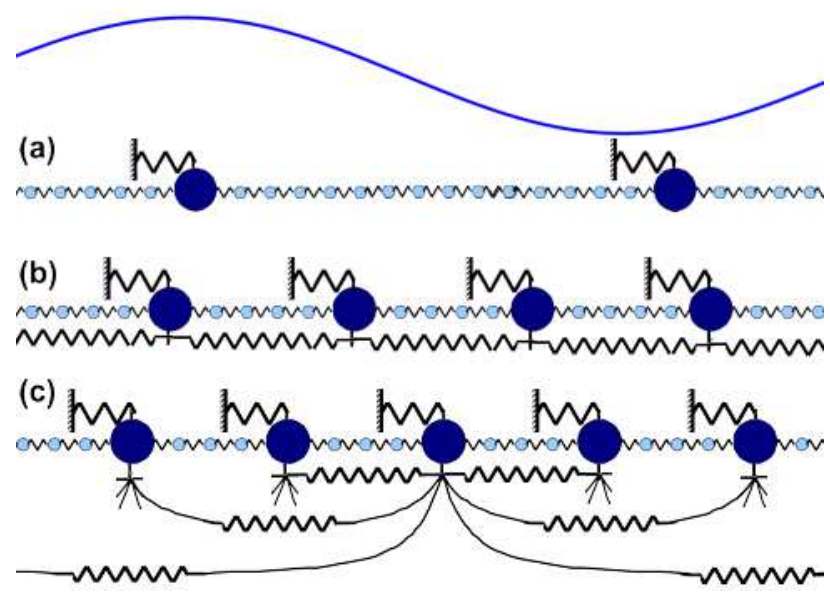

FIG. 7: Mechanical analogy of the metamaterial in the photonic crystal regime (a), homogeneous (b) and inhomogeneous (c) medium. Small balls and springs represent host medium. Large balls represent resonant inclusions.

nance frequency. When the distance between the metaatoms is large, so that $\Re(k a)>\pi$, the periodic medium is in the photonic crystal regime (Fig. $7 \mathrm{~h}$ ). The massive oscillators periodically arranged in the system scatter the wave individually. Then constructive or destructive interference leads to the formation of stop-bands and pass-bands. When the distance between meta-atoms decreases, but coupling between monolayers is still weak, we get a kind of coupled resonator waveguide, where only nearest-neighbor coupling matters (Fig. 7b). Such a waveguide has one fundamental mode (two in case of chiral metamaterials), which is dominating the light propagation. Thus the periodic medium may be homogenized, and the extraordinary properties of MMs originate from the meta-atom resonances. When the distance between the meta-atoms is further reduced, the coupling gets stronger and the nearest-neighbor scenario ceases to hold. Now higher-order modes come into play (Fig. 7 F) and participate in the energy transfer together with the fundamental mode. So, this is the regime, when higherorder Bloch modes can propagate in the structure, and homogenization is not feasible.

As can be inferred from the spring-ball model the effect of higher-order modes is negligible if there is no strong interaction between the meta-atoms. In this case the effective properties are solely determined by the resonances of the individual meta-atoms. However, to ensure a decoupling while the period has to remain small in comparison to the wavelength, i.e., avoiding photonic crystal effects, the constitutive elements of the meta-atoms have to be very small. Obviously, in this case the resonance features of the meta-atoms disappear for a fixed frequency. Such MM would hardly exhibit any extraordinary properties. 


\section{CONCLUSIONS}

To sum up, it turns out that the possibility of homogenization of chiral MM depends crucially on the coupling strength between meta-atoms. For weak coupling between monolayers the MM can be homogenized. Then the optimal meta-atom separation can be identified by looking at the Bloch mode dispersion relation in the frequency domain below the Bragg resonance. The TC MM homogenization works best if the characteristic parameter $a$ of the unit cell is as small as possible but the higherorder Bloch modes remain well separated from the fundamental modes. In this contribution we propose the analytical homogenization criterion as $\Delta \Im(k a)>0.5$. In this respect it is helpful that the calculation of the dispersion relation for two unit cell parameters $a$ suffices to estimate the lower homogeneity limit of a metamaterial.

For strong coupling between meta-atoms, the homoge- nization of MMs might be impossible at all. In this case the functionality of a MM, e.g. a stack of several monolayers, must be characterized rather than retrieving EPs of one monolayer and extrapolating them to the properties of a multilayered stack.

\section{Acknowledgments}

The authors wish to thank M.Wubs for critical comments on the manuscript. A.A., R.M. and A.L. acknowledge financial support from the Danish Research Council for Technology and Production Sciences via the NIMbus project and by COST Action MP0702. C.M., C.R. and F.L. acknowledge financial support by the German Federal Ministry of Education and Research (Metamat, PhoNa) and by the Thuringian State Government (MeMa).

* andra@fotonik.dtu.dk

1 M. Decker, M. Ruther, C. E. Kriegler, J. Zhou, C. M. Soukoulis, S. Linden, and M. Wegener, Opt. Lett. 34, 2501 (2009).

2 E. Plum, V. A. Fedotov, A. S. Schwanecke, N. I. Zheludev, and Y. Chen, Appl. Phys. Lett. 90, 223113 (2007).

3 A. V. Rogacheva, V. A. Fedotov, A. S. Schwanecke, and N. I. Zheludev, Phys. Rev. Lett. 97, 177401 (2006).

4 M. Decker, M. W. Klein, M. Wegener, and S. Linden, Opt. Lett. 32, 856 (2007)

5 J. K. Gansel, M. Thiel, M. S. Rill, M. Decker, K. Bade, V. Saile, G. von Freymann, S. Linden, and M. Wegener, Science 325, 1513 (2009).

${ }^{6}$ D. Kwon, P. L. Werner, and D. H. Werner, Opt. Express 16, 11802 (2008).

7 S. Tretyakov, I. Nefedov, A. Sihvola, S. Maslovski, and C. Simovski, J. Electr. Magn. Wav. and Appl. 17, 695 (2003).

8 J. B. Pendry, Science 306, 1353 (2004).

9 J. Zhou, J. Dong, B. Wang, T. Koschny, M. Kafesaki, and C. M. Soukoulis, Phys. Rev. B 79, 121104 (2009).

10 B. Wang, J. Zhou, T. Koschny, and C. M. Soukoulis, Appl. Phys. Lett. 94, 151112 (2009).

11 S. Zhang, Y.-S. Park, J. Li, X. Lu, W. Zhang, and X. Zhang, Phys. Rev. Lett. 102, 023901 (2009).

12 M. C. K. Wiltshire, J. B. Pendry, and J. V. Hajnal, J. Phys. Cond. Matt. 21, 292201 (2009).

13 D. R. Smith, S. Schultz, P. Markos, and C. M. Soukoulis, Phys. Rev. B 65, 195104 (2002).

14 C. Menzel, C. Rockstuhl, T. Paul, and F. Lederer, Appl. Phys. Lett. 93, 233106 (2008).

15 E. Plum, J. Zhou, J. Dong, V. A. Fedotov, T. Koschny, C. M. Soukoulis, and N. I. Zheludev, Phys. Rev. B 79, 035407 (2009).

16 B. Wang, J. Zhou, T. Koschny, M. Kafesaki, and C. M. Soukoulis, J. Opt. A 11, 114003 (2009).

17 X. Xiong, W.-H. Sun, Y.-J. Bao, M. Wang, R.-W. Peng, C. Sun, X. Lu, J. Shao, Z.-F. Li, and N.-B. Ming, Phys. Rev. B 81, 075119 (2010).

18 C. Simovski, Opt. Spectrosc. 107, 726 (2009).

19 J. Zhou, T. Koschny, M. Kafesaki, and C. M. Soukoulis, Phys. Rev. B 80, 035109 (2009).

20 C. R. Simovski, Metamaterials 1, 62 (2007).

21 A. Andryieuski, C. Menzel, C. Rockstuhl, R. Malureanu, and A. V. Lavrinenko, J. of Opt. A 11, 114010 (2009).

22 N. Liu and H. Giessen, Opt. Express 16, 21233 (2008).

23 I. Sersic, M. Frimmer, E. Verhagen, and A. F. Koenderink, Phys. Rev. Lett. 103, 213902 (2009).

${ }^{24}$ K. Aydin, I. Pryce, and H. Atwater, Opt. Express 18, 13407 (2010).

25 E. Shamonina and L. Solymar, J. Magn. and Magn. Mat. 300, 38 (2006).

26 H. Liu, Y. M. Liu, T. Li, S. M. Wang, S. N. Zhu, and X. Zhang, Phys. Status Solidi B 246, 1397 (2009).

27 Z. Li, H. Caglayan, E. Colak, J. Zhou, C. M. Soukoulis, and E. Ozbay, Opt. Express 18, 5375 (2010).

28 C. Menzel, C. Rockstuhl, T. Paul, F. Lederer, and T. Pertsch, Phys. Rev. B 77, 195328 (2008).

29 C. Menzel, C. Rockstuhl, R. Iliew, F. Lederer, A. Andryieuski, R. Malureanu, and A. V. Lavrinenko, Phys. Rev. B 81, 195123 (2010).

30 C. Rockstuhl, T. Paul, F. Lederer, T. Pertsch, T. Zentgraf, T. P. Meyrath, and H. Giessen, Phys. Rev. B 77, 035126 (2008).

31 J. Yang, C. Sauvan, T. Paul, C. Rockstuhl, F. Lederer, and P. Lalanne, Appl. Phys. Lett. 97, 061102 (2010).

32 N. A. Mortensen, M. Yan, O. Sigmund, and O. Breinbjerg, J. Eur. Opt. Soc. 5, 10010 (2010).

33 M. Decker, R. Zhao, C. M. Soukoulis, S. Linden, and M. Wegener, Opt. Lett. 35, 1593 (2010).

34 CST computer simulation technology AG, http://cst.com/.

35 A. Andryieuski, R. Malureanu, and A. V. Lavrinenko, Phys. Rev. B 80, 193101 (2009).

36 A. Andryieuski, R. Malureanu, and A. V. Lavrinenko, Opt. Express 18, 15498 (2010).

37 Z.-Y. Li and L.-L. Lin, Phys. Rev. E 67, 046607 (2003).

38 C. Rockstuhl, C. Menzel, T. Paul, T. Pertsch, and F. Lederer, Phys. Rev. B 78, 155102 (2008).

39 K. J. Boller, A. Imamoglu, and S. E. Harris, Phys. Rev. Lett. 66, 2593 (1991). 
${ }^{40}$ N. Liu, T. Weiss, M. Mesch, L. Langguth, U. Eigenthaler, M. Hirscher, C. Sonnichsen, and H. Giessen, Nano Lett. 10, 1103 (2010).
41 L. Brillouin, Wave propagation in periodic structures (Dover Pubns, 2003). 